\title{
Conjugate observations of the day-side reconnection electric field: A GEM boundary layer campaign
}

\author{
M. Pinnock ${ }^{1}$, A. S. Rodger ${ }^{1}$, K. B. Baker ${ }^{2}$, G. Lu $^{3}$, M. Hairston ${ }^{4}$ \\ ${ }^{1}$ British Antarctic Survey, Natural Environment Research Council, Cambridge, UK \\ 2 The Johns Hopkins University, Applied Physics Laboratory, Laurel, Maryland, USA \\ ${ }^{3}$ High Altitude Observatory, National Center for Atmospheric Research, Boulder, Colorado, USA \\ ${ }^{4}$ Center for Space Sciences, Univ. of Texas at Dallas, Richardson, Texas, USA
}

Received: 2 June 1998 / Revised: 22 September 1998 / Accepted: 25 September 1998

\begin{abstract}
Data from HF-radars are used to make the first simultaneous conjugate measurements of the dayside reconnection electric field. A period of $4 \mathrm{~h}$ around local magnetic noon are studied during a geospace environment modeling (GEM) boundary layer campaign. The interplanetary magnetic field (IMF) was southward whilst the eastward component (By) was variable. The flow patterns derived from the radar data show the expected conjugate asymmetries associated with IMF $|\mathrm{By}|>0$. High-time resolution data (50 and $100 \mathrm{~s}$ ) enable the flow of plasma across the open/closed field line boundary (the separatrix) to be studied in greater detail than in previous work. The latitude of the separatrix follows the same general trend in both hemispheres but shows a hemispherical difference of $4^{\circ}$, with the summer cusp at higher latitude, as expected from dipole tilt considerations. However, the short-time scale motion of the separatrix cannot be satisfactorily resolved within the best resolution $\left(300 \mathrm{~m} \mathrm{~s}^{-1}\right)$ of the experiment. The orientation of the separatrix with respect to magnetic latitude is found to follow the same trend in both hemispheres and qualitatively fits that predicted by a model auroral oval. It shows no correlation with IMF By. However, the degree of tilt in the Northern (summer) Hemisphere is found to be significantly greater than that given by the model oval. The convection pattern data show that the meridian at which throat flow occurs is different in the two hemispheres and is controlled by IMF By, in agreement with empirically derived convection patterns and theoretical models. The day-side reconnection electric field values are largest when the radar's meridian is in the throat flow or early afternoon flow regions. In the morning or afternoon convection cells, the reconnection electric field tends to zero away from the throat flow region. The reconnection electric field observed in the throat flow region is bursty in nature.
\end{abstract}

Correspondence to: M. Pinnock

E-mail: M.Pinnock@bas.ac.uk
Key words. Ionosphere (plasma convection; polar ionosphere) - Magnetospheric physics (magnetosphereionosphere interactions).

\section{Introduction}

Magnetic reconnection on the day-side magnetopause is the dominant mechanism by which solar wind energy is transferred to the Earth's magnetosphere, at least when the interplanetary magnetic field (IMF) has a southward component. Determining the reconnection rate on the day-side, together with its counterpart on the night-side in the magnetotail, is therefore of primary importance in describing the dynamics of the magnetosphere/ionosphere coupled system.

The magnetopause reconnection line (or active $X$ line) maps in to a small area of the ionospheric cusp (e.g., Crooker and Toffoletto, 1995), and thus presents the opportunity to determine the day-side reconnection rate through ionospheric measurements. To do this one must measure the instantaneous voltage applied across the day-side merging gap (Siscoe and Huang, 1985), which by Faraday's law equates to the magnetic flux being added to the polar cap (Coroniti and Kennel, 1973). This requires the rate at which plasma flows across the open/closed magnetic field line boundary, in the rest frame of the boundary, to be determined along the full length of the ionospheric footprint of the magnetopause reconnection line.

Unfortunately the length of the reconnection line in the ionosphere is longer than the field-of-view of any single instrument, so it is only possible to measure a segment of the reconnection electric field. In this study, we employ HF radars to determine the instantaneous day-side reconnection electric field $\left(\mathrm{E}_{r e c}\right)$ on nearly conjugate meridians, in the ionosphere. Conjugate measurements of $\mathrm{E}_{\text {rec }}$ can provide two independent measurements which, to a first order should be equal. 
However, when the contribution from an IMF east-west component (By) are considered at least two asymmetries may arise which lead to differing values of $\mathrm{E}_{\text {rec }}$ being measured in each hemisphere. The first asymmetry is due to the longitudinal shift of the cusp, and hence the "throat flow" region which entrains most of the flux entering the polar cap from the day-side. This has been illustrated experimentally (Heppner and Maynard, 1987; Newell et al., 1989) and described theoretically by Cowley et al. (1991). The second asymmetry arises from the predictions of the antiparallel merging theory put forward by Crooker (1979). Consideration of the vector motion of newly reconnected flux tubes at different points along the merging line predicts that the magnitude of the flows should vary across the ionospheric merging gap.

The motion of the open/closed field line boundary (hereafter referred to as the separatrix) is a key component in deriving the reconnection voltage (e.g., Pudovkin et al., 1992). Because the flow in the rest frame of the separatrix is used to derive $\mathrm{E}_{\text {rec }}$, when the separatrix is moving equatorward its velocity adds to the poleward flow velocity component. Using meridian scanning photometer data taken at $630 \mathrm{~nm}$, Pudovkin et al. (1992) claimed that equatorward motions occur over time scales of $3 \mathrm{~min}$ and distances between $0.5^{\circ}$ and $2^{\circ}$, which gives separatrix velocities of between 400 and $2600 \mathrm{~m} \mathrm{~s}^{-1}$. Lockwood et al. (1993a) have estimated an equatorward velocity of the separatrix of $670 \mathrm{~m} \mathrm{~s}^{-1}$. These values compare with typical poleward flow components in the cusp ionosphere of the order of $500-1000 \mathrm{~m} \mathrm{~s}^{-1}$, with occasional enhancements to $\sim 2000 \mathrm{~m} \mathrm{~s}^{-1}$ resulting from flow channel events (e.g., Pinnock et al., 1993).

There have been several proxies used for the identification of the ionospheric signature of the separatrix. These include the equatorward edge of the low latitude boundary layer precipitation near noon (Lockwood et al., 1993b), the equatorward edge of the $630 \mathrm{~nm}$ emission (Moen et al., 1996) and the equatorward edge of the turbulent plasma regime detected by HF radar (Baker et al., 1995). Each of these proxies has limitations (Minow, 1996; Rodger, 1998) but the HF radar method provides the best spatial and temporal resolution and thus is the proxy we adopted. Its greatest limitation is that it is $\sim 100 \mathrm{~km}$ poleward of the true separatrix position (Rodger and Pinnock, 1997) but its motion is expected to mimic that of the separatrix.

This study builds on the earlier work reported by Baker et al. (1997), using a single Northern Hemisphere radar. The averaging technique, over $5 \mathrm{~min}$ time periods, used in the earlier study is quite complex and its consequences with respect to cusp transients are not easy to determine. There is thus merit in using data with greater resolution, as noted in Baker et al. (1997). The method for deriving $\mathrm{E}_{\text {rec }}$ is similar to that used by Baker et al. (1997). Our aim is to make conjugate comparisons, so the reconnection electric field is derived for nearly conjugate magnetic meridians, rather than the potential difference across the whole radar field-of-view as was done in Baker et al.(1997). Note however, that because single radars are being used the limitations imposed by plasma flow vectors derived from the beam-swinging technique still apply. One further limitation is that we have no knowledge of the night-side reconnection rate during the study period. The size of the polar cap, and hence the latitude of the separatrix, is controlled by both the day-side and the night-side reconnection rates (Siscoe and Huang, 1985).

\section{Experimental technique}

Data from the PACE HF radars (Baker et. al., 1989) at Goose Bay, Labrador $\left(53^{\circ} \mathrm{N}, 60^{\circ} \mathrm{W}\right)$ and Halley, Antarctica $\left(76^{\circ} \mathrm{S}, 27^{\circ} \mathrm{W}\right)$ are used to study the period 1300-1700 UT, 21 July, 1992. This was a geospace environment modeling (GEM) campaign period. The radars were operating in normal scanning mode, sweeping a single beam of width $\sim 4^{\circ}$ through an azimuth of $52^{\circ}$. The Halley radar was operating with an integration time on each beam of $6 \mathrm{~s}$, one scan being completed in $100 \mathrm{~s}$ after allowance for radar set up time. The Goose Bay radar was operated with two integration periods on this day, 6 and $3 \mathrm{~s}$, giving a scan resolution of 100 and $50 \mathrm{~s}$ respectively. Some data gaps occur due to operator intervention to change the radar's programme. The Halley radar was operated with a range gate of $45 \mathrm{~km}$, the Goose Bay radar was operating with a range gate of $30 \mathrm{~km}$.

In this study, the AACGM coordinate system is used, a development of the PACE geomagnetic coordinate system of Baker and Wing (1989).

Only F-region backscatter, which drifts with the ambient plasma drift (Villain et al., 1985), has been used in this study. The scatter from all beam positions are used to derive 2-D velocity vectors on the radar beam closest to the magnetic meridian, using the sophisticated beam-swinging technique described by Ruohoniemi et al., (1989). The plasma vector on the magnetic meridian that is closest to the latitude of the separatrix is taken to represent the flow at the separatrix. For the Halley radar, the meridian is at $30^{\circ} \mathrm{E}$ geomagnetic $(\mathrm{MLT}=\mathrm{UT}-3.5 \mathrm{~h})$, whilst the Goose Bay meridian is $23^{\circ} \mathrm{E}(\mathrm{MLT}=\mathrm{UT}-4 \mathrm{~h})$. The flow across the separatrix takes account of the orientation of the separatrix.

The location of the boundary between low and high Doppler spectral widths in the radar data is used to determine the position of the separatrix, see Baker et al. (1995) for a description and verification of this technique. Note also that this technique has been calibrated against the $630 \mathrm{~nm}$ cusp emission (Rodger et al., 1995). The separatrix is determined with the same criteria used by Baker et al. (1997); the range gate at which the Doppler spectral width exceeds $150 \mathrm{~m} \mathrm{~s}^{-1}$. Where possible three points are scaled from each radar scan map, one each on the western and eastern edges of the radar scatter and another at $30^{\circ} \mathrm{E}$ geomagnetic. The tilt angle of the separatrix, with respect to a line of geomagnetic latitude, is calculated from these three points using a straight line fit. The latitude of the separatrix at $30^{\circ} \mathrm{E}$ is 
used as the reference in determining the motion of the separatrix and also to enable conjugate comparisons to be made. This procedure differs from that used by Baker et al. (1997), who scaled the separatrix latitude in each of the 16 beams and then fitted a smoothed curve to the data points. Our simpler technique, employed because of the much larger data quantities involved in this study, will smooth out any small scale features (e.g., a few hundred kilometres) on the separatrix. Very few such features were found in the data.

\subsection{Errors and uncertainties}

2.1.1 Vector velocity determination. In determining the velocity vectors, line-of-sight Doppler velocities with a measurement error greater than $50 \mathrm{~m} \mathrm{~s}^{-1}$ were rejected. To produce a vector at a particular latitude a minimum of data from 5 beams was required and the rms error of the cosine fitting process had to be less than $50 \mathrm{~m} \mathrm{~s}^{-1}$. Possible errors in vectors derived by the beam swinging technique of Ruohoniemi et al. (1989) have been discussed fully by Freeman et al. (1991). However, as we are interested in a single velocity component, the flow crossing the separatrix, we can check the derived velocities using the line-of-sight velocity data on the beam closest to orthogonal to the separatrix. This check is not always possible, large separatrix tilt angles may mean that no beam is orthogonal. The check has been performed for several samples of the data and shows good agreement (within the error of the line-of-sight velocity determination, $<50 \mathrm{~m} \mathrm{~s}^{-1}$ ) between the velocity component derived from beam swinging and the line-ofsight velocity data.

The beam-swinging technique reproduces well steady flows but will fail to resolve flows which are varying on time scales comparable to the scan time (50 or $100 \mathrm{~s}$ ) and/or on spatial scales of less than $400 \mathrm{~km}$. The cusp is known to contain rapidly varying features, e.g., flow bursts resulting from flux transfer events (FTEs) (Pinnock et al., 1993). Under such conditions the technique usually fails to produce vectors, giving data gaps. Baker et al. (1997) argued that the underestimation of the reconnection voltage arising from such transients is of the order of $3 \mathrm{kV}$ potential difference.

2.1.2 Determining the latitude of the separatrix. The accuracy of location of HF radar backscatter has been estimated to be $\pm 20 \mathrm{~km}$ by experimental and raytracing techniques (Andre et al., 1997 and references therein) and to within \pm 1 range gate when comparing geophysical parameters measured by HF radar and other instruments (e.g., Baker et al., 1990). We therefore take it that the location of the separatrix can be determined with an accuracy of one range gate; $45 \mathrm{~km} / 0.4^{\circ}$ latitude for Halley, $30 \mathrm{~km} / 0.27^{\circ}$ latitude for Goose Bay. Note that variations of one range gate do not necessarily imply boundary movement of $30 / 45 \mathrm{~km}$. Movement of an echoing region across a range gate boundary will produce an apparent shift of 45 or $30 \mathrm{~km}$.
The Doppler spectral width measured by the radar may be susceptible to variations in HF radio noise. To guard against this, the determination of the separatrix latitude was done using a high signal/noise ratio ( $\geq 9 \mathrm{~dB})$ as a threshold for the data. As a further check, we have visually compared time series of the backscattered power in the vicinity of the separatrix latitude and the separatrix latitude. No correlation between these two time series was found.

The DMSP satellite orbits available for this period do not encounter the cusp, and so cannot be used for independently verifying the separatrix latitude near noon.

2.1.3 Determining the tilt of the separatrix. The determination of the tilt of the separatrix depends on the measurement accuracy described in sect. (2.1.2) and the longitudinal span separating the two outermost measurements of the separatrix latitude. For Goose Bay data the standard error is typically $2.5^{\circ}$, for Halley data it is $5.8^{\circ}$.

2.1.4 Determining the reconnection electric field. The error on the flow crossing the separatrix is largest when the plasma flow direction is nearly parallel to the separatrix and a minimum when the flow is orthogonal to the separatrix. Typical errors are a few tens of $\mathrm{m} \mathrm{s}^{-1}$ for both radars but can reach values between 100 $200 \mathrm{~m} \mathrm{~s}^{-1}$ in the afternoon convection cell.

\section{Observations}

\subsection{Background conditions}

Solar wind and IMF data from IMP-8 for this period are shown in Fig. 1. The IMF turned southward at $\sim 1153$ UT and remained so throughout the period studied. At the start of the period (1300 UT) IMF By is negative, turning positive at $1345 \mathrm{UT}$. It remains positive until 1520 UT when it goes to zero and then negative at 1531 UT. From 1540-1700 UT, it is positive except for one brief By negative period at $\sim 1630$ UT. IMF $\mathrm{Bx}$ is positive throughout (typically between +5 and $+10 \mathrm{nT}$ ) except for the last $15 \mathrm{~min}$ when it goes negative.

The solar wind dynamic pressure $\left(\mathrm{nm} \mathrm{v}^{2}\right.$, Fig. 1e) for this period is high, typically of the order 6-7 nanoPascals, and its variability arises almost solely from the density variations. The solar wind particle density is typically in the range 45 to 35 particles $\mathrm{c} \mathrm{c}^{-3}$ whilst the velocity is stable at about $310 \mathrm{~km} \mathrm{~s}^{-1}$, varying by only $\pm 10 \mathrm{~km} \mathrm{~s}^{-1}$.

The plasma vectors derived from the Halley data around the interval of the By change at 1345 UT have been analyzed in the manner of Greenwald et al. (1990) and show that the delay from the satellite sensing the IMF By change to the convection pattern change seen in the ionosphere is of the order of $1 \mathrm{~min}$. This is a surprisingly short time, given the location of the satellite close to the Sun-Earth line, and implies that 

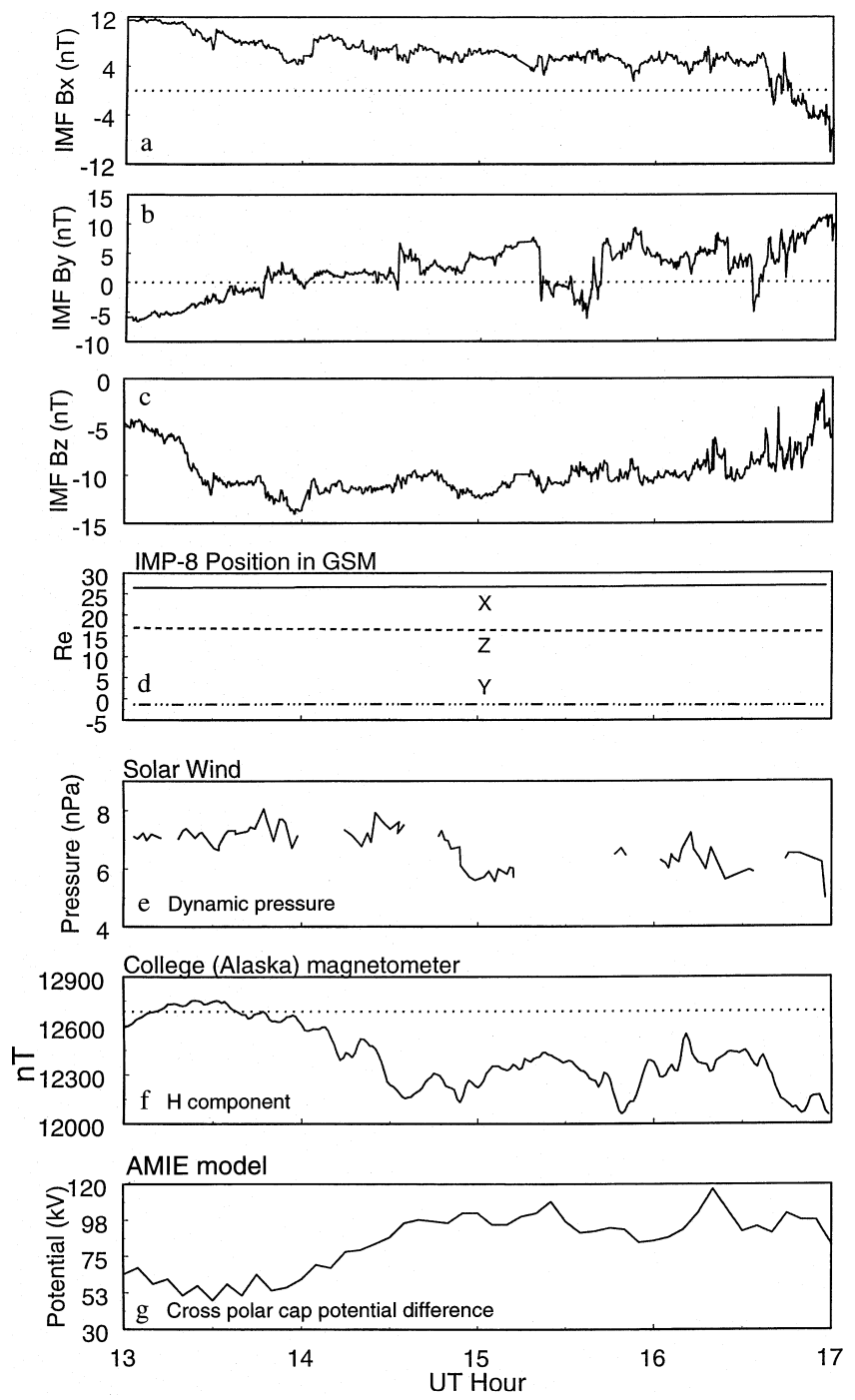

Fig. 1a-g. The interplanetary magnetic field, a Bx, b By and c Bz, $\mathbf{d}$ the position of the spacecraft and $\mathbf{e}$ the solar wind dynamic pressure for the period $1300-1700$ UT on 21 July, 1992 measured by the IMP-8 spacecraft. $\mathbf{f}$ The College (Alaska) magnetogram H component (solid line) and its baseline value (dashed line). $\mathbf{g}$ The total cross polar cap potential difference (CPD) in the Northern Hemisphere derived from the AMIE modeling process

the wave front in the solar wind is not aligned as a Parker spiral.

The College (Alaska) magnetogram $\mathrm{H}$ trace (Fig. 1f) shows how our measurements relate to the substorm cycle. At 1400 UT, College is at $\sim 0215$ MLT in the AACGM coordinate system. From 1300-1410 UT, the magnetogram shows only minor perturbations from the baseline value, with possibly a growth phase developing from $1340 \mathrm{UT}$ onwards. AT $1410 \mathrm{UT}$ a sharp (160 nT) negative bay onset is seen followed by continuing activity over the next $4 \mathrm{~h}$. We interpret this as a substorm onset being initiated close to 1410 UT and with multiple substorm onsets thereafter.

The AMIE model (Richmond and Kamide, 1988) has been run for this period, taking as input data from 78 magnetometer stations, the Goose Bay radar and
DMSP satellite data. In this work, we use the AMIE output to assess the likely cross-polar cap potential difference (CPD). The bottom panel in Fig. 1 shows the time variation of the CPD for the Northern Hemisphere. At the start of the study period the CPD is $\sim 60 \mathrm{kV}$ and after $1400 \mathrm{UT}$ rises to around $100 \mathrm{kV}$, where it remains for the rest of the interval.

An overview of the plasma flow conditions, with vectors averaged over $1^{\circ}$ of latitude and 10 min intervals for each hemisphere, is shown in Fig. 2. The Northern Hemisphere data (top panel) starts (1300 UT, 0900 MLT) with predominantly poleward flows (the throat region) being observed and then after the IMF By change at 1345 UT, the afternoon cell is observed. In the Southern Hemisphere, at the start of the interval the morning convection cell return flow is observed. After the By change at 1345 UT, the flows have the characteristics of the early afternoon convection cell. The afternoon cell continues to be observed for the remainder of the study period.

In this study, we are concerned with the vectors at the latitude of the separatrix, which are close to the equatorward edge of the vectors shown in Fig 2. However, the hemispheric asymmetries in flow at higher latitudes expected from IMF By effects (e.g., Greenwald

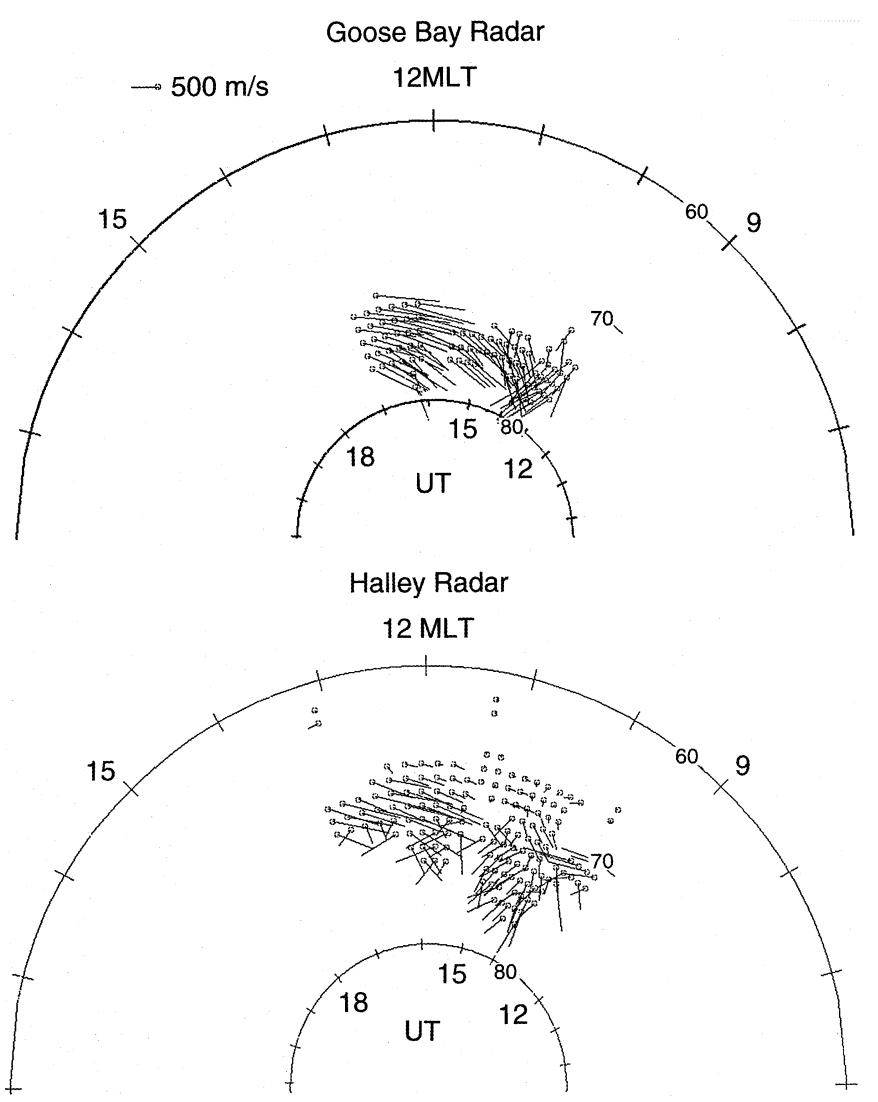

Fig. 2. A summary of the flow conditions observed in the two hemispheres. Vectors averaged over 10 min land $1^{\circ}$ of latitude are shown. For each vector the circle marks the point at which the measurement was made and the line the magnitude and direction of the vector. Latitude is in AAGCM magnetic coordinates. The outer circle $\left(60^{\circ}\right.$ latitude) is marked in Magnetic Local Time, the inner circle ( $80^{\circ}$ latitude) is in Universal Time 
et al., 1990) are seen in the data. For example, at 1600 UT, the Goose Bay high latitude flows have a dawnward component, whilst at Halley they are duskward which is consistent with the prevailing IMF By positive conditions.

\subsection{The separatrix}

The characteristics of the separatrix, using data at $100 \mathrm{~s}$ and $50 \mathrm{~s}$ resolution, are shown in Fig. 3a for Goose Bay and in Fig. 3b for Halley, note that the y-axes are not scaled the same in each figure. The latitude of the separatrix at $30^{\circ} \mathrm{E}$ (top panel) shows the same equatorward trend in both hemispheres, however, there are detailed differences. From 1300-1410 UT, the latitude of the separatrix is relatively constant in both hemispheres (taking into account the error bars). After 1410 UT, the Northern Hemisphere results show an equatorward shift $\left(0.75^{\circ}\right)$ of the separatrix, which then remains at constant latitude until 1500 UT. No such equatorward motion is seen in the Southern Hemisphere, but the larger error limits on this data set would prevent detection of a shift of comparable magnitude to that seen in the north. There is an interval just after 1500 UT when there is a poleward shift in both hemispheres, with a return to equatorward motion by 1530 UT. From 1630-1700 UT, the Halley data show no overall motion whilst the Goose Bay data show a continuing equatorward trend. The latitude of the separatrix in both hemispheres shows short-lived equatorward and poleward excursions superposed on the general trend but these are normally within the uncertainties of measurement.

From 1300-1500 UT, the average separatrix latitude is $-71.0^{\circ}$ for Halley and $74.8^{\circ}$ for Goose Bay. The Northern (summer) Hemisphere separatrix is consistently 3 to $4^{\circ}$ higher in latitude except for the period from 1630-1700 UT when the difference steadily reduces to $\sim 2^{\circ}$.

Figure $3 a(b)$ and $3 b(b)$ shows the tilt of the separatrix (solid line) from alignment with a line of geomagnetic latitude. The dashed line shows the tilt, over one hour of MLT, calculated for the locus of the poleward edge of the Feldstein (1963) auroral oval, as given by the equations of Holzworth and Meng (1975). We would expect the separatrix to be closely associated with the poleward edge of the oval. The model for $\mathrm{Q}=6$ is required to give a latitude comparable with the data for the separatrix in the Northern Hemisphere. From the model oval the largest tilts observed are $0.8^{\circ}$ of latitudinal difference over one hour, which corresponds to a tilt angle of $10^{\circ}$ at the relevant MLTs. The model oval fits the Halley data very well, with the data having an average $\sim 5^{\circ}$ (which is within the standard error) more positive tilt than the model. The Goose Bay data also fit the model reasonably well except between 1320 1340 UT, 1455-1535 UT and after 1630 UT. It is interesting to note that the poor fits to the model occur when the rate of change of the IMF (Bz and/or By, see Fig. 1) is greatest.
The meridional velocity of the separatrix is shown in panel (c), derived from consecutive determinations of the latitude of the separatrix at $30^{\circ} \mathrm{E}$. Also shown in this plot are the uncertainties associated with a movement of \pm 1 range gate within the sampling time. The vast majority of separatrix velocities are within the error limits, in the case of Goose Bay (Fig. 3a, c) the uncertainty limits are only exceeded on five occasions out of the 139 measurements whilst for Halley (Fig 3b, c) it is two occasions out of 148 measurements. It is clear that the experimental resolution has not been sufficient to accurately resolve the short-time scale (50 and $100 \mathrm{~s}$ ) movement of the separatrix for most of the study period, although we can set some upper limits. By implication, most of the separatrix movement is contained within one range gate $(30$ or $45 \mathrm{~km})$ over the sampling period. To include this separatrix velocity in the calculation of the reconnection voltage would add noise and therefore it is not included in our calculation. However, in order to include the general trend of the separatrix in the derivation of the flow across the separatrix, the data have been smoothed (using a Lowess locally weighted regression, smoothing algorithm, bold line in Fig. 3a, c and Fig. 3b, c) and these smoothed values are used in the calculation. The consequences of this for the accuracy of our reconnection voltage data are discussed in Sect. 4.

\subsection{Flow across the separatrix}

In Fig. 3 panels (d) and (e) summarise the flow conditions observed at each radar in terms of the magnitude and direction. We use the term "throat flows" to mean regions where the poleward flow component dominates (average angle of flow between 45 and $135^{\circ}, 90^{\circ}$ is poleward). Panel (f) shows the magnitude of the flow across the separatrix in the rest frame of the separatrix (using the smoothed separatrix velocity values) at the radar's magnetic meridian. The right hand $y$-axis of panel (f) converts this flow to an electric field value, assuming $\mathrm{E} \times \mathrm{B}$ drift and an ionospheric magnetic field of $50000 \mathrm{nT}$. This electric field value is the day-side reconnection electric field $\left(\mathrm{E}_{\text {rec }}\right)$ measured on the magnetic meridian.

If it is assumed that the total day-side reconnection electric field is applied in a uniform fashion across a fixed range of MLT, then the $\mathrm{E}_{r e c}$ measured at a particular meridian will vary as the arc length of the separatrix in the ionosphere varies with the latitude of the separatrix. To illustrate, if the Halley data are taken at $-70^{\circ}$ and the Goose Bay data at $75^{\circ}$ latitude, then the Halley $\mathrm{E}_{\text {rec }}$ would be $75 \%$ of the Goose Bay value. No correction is made in the data for this geometric factor.

The data show that basically three flow regimes are sampled by the radars:

a. The morning cell return flow, 1300-1345 UT (09301015 MLT) by the Halley radar only. The plasma flow direction shows little variability.

b. The afternoon cell return flow, $\sim 1600$ to 1700 UT (1230-1330 MLT) by both radars. The plasma flow 


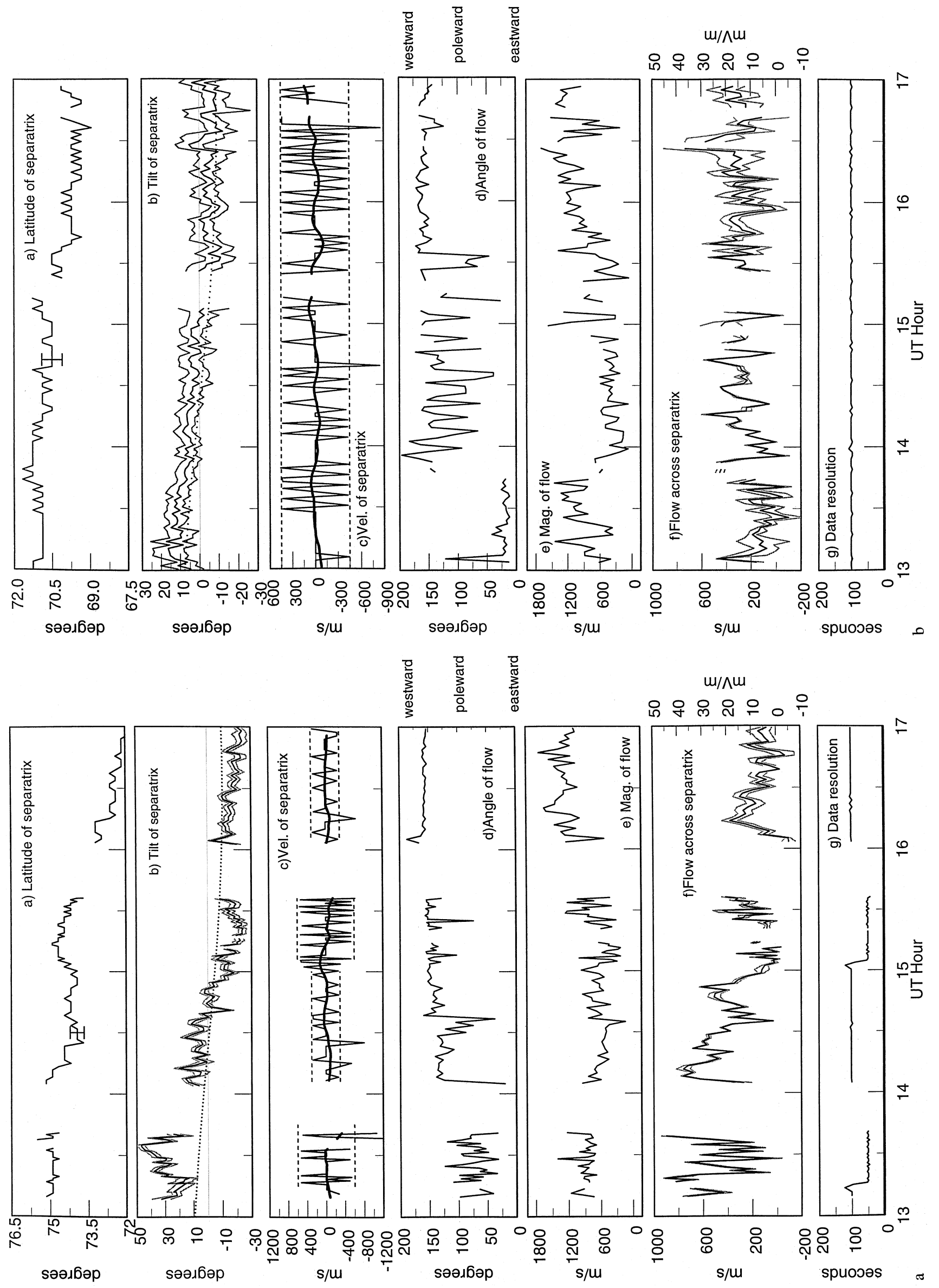


Fig. 3a. Summary of the data from the Goose Bay radar (Northern Hemisphere), taken on the $23^{\circ} \mathrm{E}$ geomagnetic meridian, except for panel a) which shows the latitude of the separatrix on the $30^{\circ} \mathrm{E}$ geomagnetic longitude. A typical error bar is shown at 1430 UT. $b$ The tilt of the separatrix away from a line of geomagnetic latitude. Positive degrees of tilt correspond to a tilt to higher latitude with later local time. Light gray lines show the upper and lower limits given by the standard error. $c$ The velocity of the separatrix (thin line). Negative values are for an equatorward acceleration of the separatrix. The bold line shows this data after smoothing by a Lowess smoothing algorithm. The horizontal dashed lines show the error limits, which vary with the time resolution of the data, see panel $g$. $d$ and $e$ The direction and magnitude of ionospheric plasma flow at the separatrix latitude, $90^{\circ}$ corresponds to poleward flow, $0^{\circ}$ and $180^{\circ}$ to eastward and westward flow respectively. $f$ The magnitude of the flow across the separatrix (left hand y-axis, positive values mean flow into the polar cap) and its equivalent electric field value at this meridian (right hand y-axis). Light gray lines show the upper and lower limits given by the standard error (see text) $\mathbf{b}$ Same as a but for the Halley radar (Southern Hemisphere), all data taken on the $30^{\circ} \mathrm{E}$ geomagnetic meridian. Note different scaling on some of the y-axes

direction shows little variability. The period 1440 1600 UT in the Goose Bay data has an average flow angle of $144^{\circ}$, and is probably best characterized as afternoon flow due to its low variability (compared to the throat region).

c. The throat flow, 1310-1340 UT (0910-0940 MLT, average flow angle $74^{\circ}$ ) and 1405-1440 UT (10051040 MLT, average flow angle $113^{\circ}$ ) in the Goose Bay data and 1350-1535 UT(1020-1205 MLT, average flow angle $123^{\circ}$ ) in the Halley data. This regime is characterized by flows which show a high degree of variability in direction from one sample to the next.

From the time of observation of the different regimes and from Fig. 2, it can be seen that for IMF By negative conditions (1300-1345 UT) the throat flow in the Northern Hemisphere is shifted to an earlier MLT than observed in the Southern Hemisphere.

Transitions in the sense of IMF By produce clear responses in the radar data. In the Halley data, Fig. $3 b(d)$, the first transition at $\sim 1345$ UT produces a change from eastward flows (morning cell) to westward flows (afternoon cell) with frequent poleward bursts. The Goose Bay radar has a data gap at this time, but from 1405 UT the data shows a swing to more westward flows (afternoon cell) but with bursts of poleward flow still continuing. The period of variable IMF By from $1520-1540$ UT is unfortunately marked by data gaps from both radars. The Halley radar has the best data coverage and does show a significant change of flow direction, swinging to a more poleward flow component for a period of $\sim 6 \mathrm{~min}$ before making a rapid return to westward flow. However, the interval $1400-1500$ UT is characterized by such swings to poleward flow and it is clear from Fig. 1 that these are not all associated with changes in the IMF By component.

Summarizing these flow conditions, the Halley radar observes the morning and afternoon cell, but throat flows are only observed during the early part of the afternoon cell (1350-1535 UT). The Goose Bay radar observes the throat flows from the beginning of the period, when it may be observing the eastern limit of the morning cell (slight eastward component), until 1440 UT when it is observing the early afternoon cell. After 1440 UT it observes the afternoon cell.

In panel (f) of Fig. 3 we shows the plasma flow across the separatrix and the resulting $\mathrm{E}_{\text {rec }}$ derived at the relevant magnetic meridian. With only a few exceptions (e.g., Goose Bay, 1605 UT) the flow is always into the polar cap, as expected in the cusp region. The Goose Bay data show the highest values (typically $600 \mathrm{~m} \mathrm{~s}^{-1}$, $33 \mathrm{mV} \mathrm{m}^{-1}$ ) at the beginning of the data period (13001340 UT, 0900-0940 MLT), when the radar is observing the throat flows. These flows are also very pulsed in nature, typically varying by $400 \mathrm{~m} \mathrm{~s}^{-1}, 20 \mathrm{mV} \mathrm{m}^{-1}$ between $50 \mathrm{~s}$ scans. It is mainly the variability in the direction of plasma flow (Fig. 3a(d)) which causes the bursts of flow across the separatrix, rather than any variation in the magnitude of the flow or the tilt of the separatrix. In the period between 1400 and 1500 UT lower flow values are observed, although occasional pulses of flow reaching $600 \mathrm{~m} \mathrm{~s}^{-1}$ occur in the early afternoon cell. From 1500 UT onwards the flow across the separatrix is of the order of $100 \mathrm{~m} \mathrm{~s}^{-1}$ and, taking in to account the standard error, relatively smooth. For the last half hour the $E_{r e c}$ is very close to $0 \mathrm{mV} \mathrm{m}^{-1}$ at this meridian. Thus maximum, and bursty, flow across the separatrix is seen in throat flow region which then steadily declines as the radar field-of-view rotates in to the afternoon convection cell.

The Southern Hemisphere flow across the separatrix at the start of the interval (morning cell) is low and the $\mathrm{E}_{\text {rec }}$ close to $0 \mathrm{mV} \mathrm{m}^{-1}$. A burst of flow across the separatrix is observed around the time of the IMF By change at 1345 UT. In the early afternoon cell observations $(1350-1535$ UT) the flow across the separatrix is comparable to the Northern Hemisphere data for the same time and region, particularly if the geometric factor identified above is taken in to account. The average flow across the separatrix in the Goose Bay data for this interval is $432 \mathrm{~m} \mathrm{~s}^{-1}$ compared with $312 \mathrm{~m} \mathrm{~s}^{-1}$ in the Halley data.

The main contrast between the Halley and Goose Bay $\mathrm{E}_{\text {rec }}$ data is in the afternoon cell ( $\left.>1535 \mathrm{UT}\right)$, this is despite the fact that the plasma flow vectors for the two hemispheres are similar in this period. The baseline flow across the separatrix in the Southern Hemisphere is large, averaging $323 \mathrm{~m} \mathrm{~s}^{-1}$ between 1525 and $1700 \mathrm{UT}$, and with frequent enhancements in the flow across the separatrix. Although the standard errors are large for this interval, they do not reduce the significance of the bursts and the one at $1628 \mathrm{UT}$ gives the largest $\mathrm{E}_{\text {rec }}$ $\left(35 \mathrm{mV} \mathrm{m}^{-1}\right)$ in the Halley data. This event is associated with a very brief period of IMF By negative (Fig. 1). The bursts at 1645 and 1655 UT occur at times when IMF By and/or Bz are varying rapidly. The three bursts arise because the tilt of the separatrix is changing at these times, whilst the plasma flow angle remains relatively constant, (compare Fig. 3b, b with Fig. 3b, f). The Northern Hemisphere separatrix tilt angle shows variations of comparable magnitude to those seen in the Southern Hemisphere, but unlike the south they do not 
go to the positive angles which promote flow across the separatrix. Thus, although the plasma flow data led us to classify the Halley data after 1535 UT as afternoon cell (and not throat flow), we do in fact observe significant flow across the separatrix in this time interval.

These descriptions gave the reconnection electric field for a specific magnetic meridian. By making certain assumptions these measurements can be extrapolated to illustrate what the total reconnection electric field potential difference may be across the day-side merging gap. This allows some comparison to be made between the computed reconnection electric field and the CPD given by the AMIE model. We take the cusp width (merging line extent) to be $3.5 \mathrm{~h}$ of MLT (Crooker and Toffoletto, 1995) and assume that the derived $\mathrm{E}_{\text {rec }}$ value is uniform across that MLT range. This last assumption may be introducing the largest errors (Crooker, 1979). Figure 4 illustrates the results from this extrapolation of the Goose Bay (Fig. 3a, f) and Halley (Fig. 3b, f) data, the dotted line in each panel is the AMIE CPD value. The Goose Bay radar observes the largest potentials in the throat flow region, with peak potentials reaching the CPD or on occasion exceeding it. In the early afternoon, the potential declines to some $20-40 \%$ of the CPD. In the afternoon cell, the reconnection potential observed is typically $10 \%$ of the CPD, declining to around $0 \mathrm{kV}$ for the last half hour of observation. The Halley radar observations start with close to $0 \mathrm{kV}$ potential difference in the morning cell. In the early afternoon cell and then through to the later afternoon cell the Halley radar is observing typically $20 \%$ of the CPD but with some bursts of reaching $50 \%$ of the CPD.
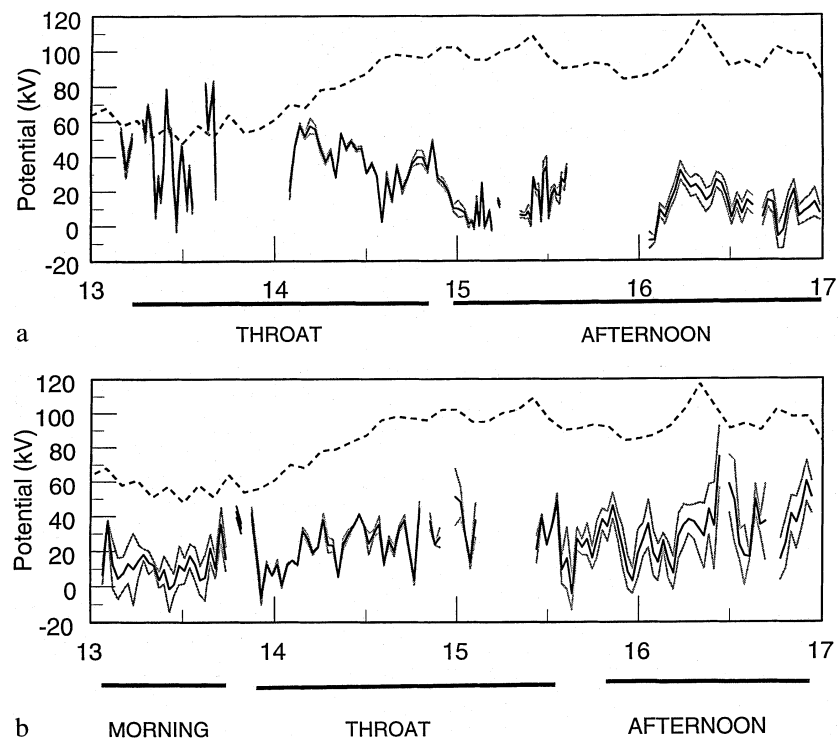

Fig. 4a, b. Time variation of the estimated potential difference (black line), assuming a magnetopause reconnection line length of $3.5 \mathrm{~h}$ of MLT, in the northern $\mathbf{a}$ and $\mathbf{b}$ southern cusp ionospheres. See text for further explanation. The light gray lines shows the upper and lower limits given by the standard error. The dashed line shows the CPD from the AMIE model, the plasma flow regimes given by the radar data are shown in bars below each figure

\section{Discussion}

\subsection{The separatrix}

The variation with time of the latitude of the separatrix is similar in both hemispheres. This has been shown in previous studies (e.g., Meng, 1982, using the equatorward edge of the cusp particle precipitation) although with a coarser time resolution given by polar orbiting satellites. The CPD derived from the AMIE model shows an increasing value through the study period, consistent with the overall equatorward motion observed.

We found an average difference in separatrix latitude, on the $30^{\circ} \mathrm{E}$ meridian in each hemisphere, in the interval $1300-1500$ UT of $3.8^{\circ}$. The dipole tilt angle at the time of these observations varies between 26 and $31^{\circ}$, close to the maximum value. Modeling work (e.g., Choe and Beard, 1974) places the summer cusp (Goose Bay observations) at $4^{\circ}$ higher latitude at the solstice. Our results are also in agreement with those of Newell and Meng (1989), who found that the low-latitude boundary layer precipitation region away from noon showed a $2^{\circ}$ conjugate asymmetry, in the same sense as is found in this study (1630-1700 UT observations). All these factors gives us confidence in our technique for determining the separatrix.

The evidence for substorm activity, from the College magnetometer, shows that the night-side convection driver could be a significant factor after 1410 UT, based on the assumption that tail reconnection is significantly enhanced at some point during the substorm expansion phase. From 1500 to 1530 UT there is an interval of poleward movement in the latitude of the separatrix, suggesting that the night-side reconnection rate may at times exceed the day-side rate during this interval.

The tilt of the separatrix in the Goose Bay data is generally larger than that observed from Halley, though the sense of the tilt is the same in both hemispheres and both change sense at $\sim 1430$ UT. The tilt angles given in Baker et al. (1997), also derived from Goose Bay radar data, are very similar in magnitude (but not sense, see later) to those presented here. The sense of the tilt is the same as that expected for the model auroral oval, but the larger values, especially in the Northern Hemisphere, suggest that other factors may be operating. These factors may include the ionospheric conductivity conditions, the southern cusp is in darkness whilst the northern cusp is illuminated (as it was for the Baker et al., 1997 study). This fact may influence the relative strengths and closure patterns of the region 0,1 and 2 field-aligned current regions, which in turn effects the mapping of the magnetic field lines between the magnetopause, magnetosphere and the ionosphere (Tsyganenko, 1991), a factor not represented in the AACGM system.

Baker et al. (1997) found that the orientation of the separatrix was correlated with IMF By, as expected from the work of Cowley et al. (1991). Our data do not support this observation, the sense of the tilt is the same in both hemispheres which is inconsistent with 
IMF By control. Furthermore, Baker et al. (1997) found that for IMF By negative conditions the tilt angle was negative, whereas we find that it is positive and for the By positive period the tilt is negative. We note that the Feldstein (1963) model is not sorted by IMF and it therefore has averaged out any IMF By effects. Another factor may be the IMF conditions, in the Baker et al. (1997) study IMF Bz was approximately comparable in value to IMF By for most of the time, for this study $\mathrm{Bz}>|\mathrm{By}|$ throughout (except for the first and last ten minutes of the study).

The factors associated with the orientation of the separatrix need further study with a much larger data set, representing a wide range of geophysical conditions.

The separatrix velocity data were included in the calculation of the reconnection voltage but only in the form of highly smoothed data, because the high time resolution data rarely exceeded the error limits. We first estimate the magnitude of the component omitted from $\mathrm{E}_{\text {rec }}$ through not including the separatrix velocity at high time resolution and then examine what conclusions can be reached regarding separatrix motion which is predominantly within our error limits.

The average equatorward motion of the separatrix during the study period, in both hemispheres, is of the order of $20 \mathrm{~m} \mathrm{~s}^{-1}$. If this is considered as an added component to the flow across the separatrix it would contribute $1 \mathrm{mV} \mathrm{m}^{-1}$ to the $\mathrm{E}_{\text {rec }}$, or $1.5 \mathrm{kV}$ of potential across $3.5 \mathrm{~h}$ of MLT. This does not effect the conclusions reached in the work. It is nevertheless an important factor when integrating the reconnection rate over time in order to compute the flux added to the polar cap.

On a small number of occasions $(<3.6 \%)$ equatorward velocities of the separatrix exceed the error limits. The data values for these occasions range from $600 \mathrm{~m} \mathrm{~s}^{-1}$ to $1200 \mathrm{~m} \mathrm{~s}^{-1}$, which would add between 30 and $60 \mathrm{mV} \mathrm{m}^{-1}$ to $\mathrm{E}_{\text {rec }}$. Their rarity means they are not a significant factor in describing the conjugate $\mathrm{E}_{\text {rec }}$.

Finally the error limits themselves, whose smallest value is $\pm 300 \mathrm{~m} \mathrm{~s}^{-1}\left(15 \mathrm{mV} \mathrm{m}^{-1}\right)$, may mask a very significant contribution to $\mathrm{E}_{\text {rec }}$. However, when the Goose Bay radar is observing the throat flow region our extrapolation (Fig. 4) gives total potentials close to $100 \%$ of the CPD determined by AMIE, which suggests that we are not missing a significant component added by the motion of the separatrix. Baker et al. (1997, see Fig. 8) also concluded that the motion of the separatrix did not add a significant component to the plasma flow across the boundary for much of the time, with a peak contribution of $20 \%$ observed in the throat flow region.

\subsubsection{Response to solar wind dynamic pressure variations.} The variability of the solar wind dynamic pressure (Fig. 1 e) influences the position of the separatrix, although observable effects may only occur for $\Delta \mathrm{p} / \mathrm{p}$ $\geq 0.5$ (Sandholt et al., 1994). We have estimated the effect of the solar wind pressure variations on our data by assuming a simple pressure balance condition and estimating the position of the magnetopause (e.g., Kivelson and Russell, 1995, p172). This is then mapped to a magnetic latitude in the ionosphere using a simple dipole model. It is found that the solar wind dynamic pressure variations shown in Fig. 1 e produce typical separatrix velocities of $\pm 100 \mathrm{~m} \mathrm{~s}^{-1}$. Maximum values, for example the pressure increase at 1425 UT (7 to $8 \mathrm{nPa}$ ), are the order of $300 \mathrm{~m} \mathrm{~s}^{-1}$. These variations are within the measurement accuracy of both radars. Comparing Fig. 1 e with $3 \mathrm{a}(\mathrm{c})$, the largest pressure pulse for which there is coincident Goose Bay data, at 1612 UT, does show a large equatorward velocity $\left(-600 \mathrm{~m} \mathrm{~s}^{-1}\right)$ followed by a poleward velocity that reaches the error limits $\left(+300 \mathrm{~m} \mathrm{~s}^{-1}\right)$. But there is no matching equatorward velocity seen at Halley at this time (error limits, $\pm 450 \mathrm{~m} \mathrm{~s}^{-1}$ ). We conclude that solar wind pressure variations for this period could give separatrix motion within our error limits. There is one pressure pulse (1612 UT) which may have produced a signature in the separatrix data from Goose Bay.

4.1.2 Response to FTEs. Models of ionospheric signature of FTEs, such as that of Cowley and Lockwood (1992), predict a specific pattern of separatrix movement which may be observable in our data (subject to measurement accuracy). This expectation is based on the same assumptions as that used by experimenters who use the $630 \mathrm{~nm}$ emission: although the $630 \mathrm{~nm}$ emissions/ high radar spectral widths are somewhat poleward of the true separatrix they are expected to mimic its behavior with time. The separatrix is expected to move equatorwards, over a relatively short time scale ( $\sim 3 \mathrm{~min}$ ), and then relax poleward over a much longer time scale $(\sim 10-15 \mathrm{~min})$ as the newly added flux is distributed within the polar cap to achieve a new equilibrium state. This picture of a single FTE signature will be modified as subsequent FTEs may occur as frequently as every 3 min (Lockwood and Wild, 1993) or even be omni-present (Pinnock et al., 1995). Under these circumstances, the equatorward movement would dominate and little poleward motion would occur. As noted in the introduction, previous reports have claimed equatorward leaps of the separatrix with velocities in the range $400-2600 \mathrm{~m} \mathrm{~s}^{-1}$. The subsequent poleward velocity of the boundary, following an isolated FTE, we estimate to be of the order of $\sim 200 \mathrm{~m} \mathrm{~s}^{-1}\left(1^{\circ}\right.$ over $10 \mathrm{~min}$ ). Thus we might expect to see the equatorward leaps in our data, if they occur, but any poleward motion of the boundary associated with FTEs would be below our measurement accuracy.

As noted earlier, we see five significant bursts of equatorward separatrix velocity in the Goose Bay data. The two largest bursts are observed in the throat flow region but others are observed in the observations of the afternoon cell. It is should also be noted that in the 35 min of throat flow observations by Goose Bay the error limits are $\pm 600 \mathrm{~m} \mathrm{~s}^{-1}$. Our observations strongly suggest that equatorward leaps of the separatrix are biased towards the lower end of the published velocity range, at $600 \mathrm{~m} \mathrm{~s}^{-1}$ or less. It is suggested that the higher velocities, such as the two events at 1000 and $1200 \mathrm{~m} \mathrm{~s}^{-1}$ 
in the Goose Bay data ( 1345 UT), may be associated with unusually large FTE events.

This has an important consequence for the evaluation of the contribution of FTEs to the CPD. Lockwood et al. (1993a) discuss how open flux may be added to the polar cap either through equatorward motion of the open/ closed field line boundary or accelerating plasma across the boundary, or a combination of both. Our findings throw the balance in favor of the observed plasma velocity being largely responsible for the addition of the flux to the polar cap. The fact that our estimates of the potential difference across the cusp region (Fig. 4) are close to $100 \%$ of the CPD, without having taken in to account the motion of the separatrix, give further support for this view.

\subsection{Flow across the separatrix and reconnection voltage}

When comparing the plasma convection data with models, it is important to consider the frame of reference. Convection patterns presented in the corotating frame (e.g., Heppner and Maynard, 1987) usually show the throat flows in the pre-noon sector for both senses of IMF By. Maynard et al. (1995) showed that when such patterns are mapped to the inertial frame of reference, the throat flows appear either side of noon, depending on IMF By. Thus our observations may be directly compared with, for example, the Heppner and Maynard (1987) convection patterns (in the corotating frame) but when comparing with the convection patterns of Cowley et al. (1991), which were presented in the inertial frame, allowance must be made for the addition of the corotation electric field.

Table 1 shows the location of the $0 \mathrm{kV}$ potential line at $70^{\circ}$ latitude in the Heppner and Maynard (1987) models DE and BC. This line is assumed to represent the centre of the throat flow region. We then assume a merging line width of $3.5 \mathrm{~h}$, centered about the $0 \mathrm{kV}$ potential line, in order to estimate the likely MLT location of the merging line (times in brackets). The table also gives the relevant radar observations for the varying IMF By conditions and time sectors.

Our data are in broad agreement with regard to the location of the ionospheric merging line as predicted by the method described. The following exceptions occur.

A.Flow across the separatrix is not predicted in the 1145-1330 MLT sector in the Southern Hemisphere during By + ve conditions but is observed.

B. Flow across the separatrix is predicted to extend to 0945 MLT, Southern Hemisphere, By -ve conditions but none is observed in the 0945-1010 MLT sector.

C. Flow across the separatrix is predicted to extend to 1315 MLT, Northern Hemisphere, By positive conditions, but is observed only until $\sim 1230$ MLT.

Exceptions $\mathrm{B}$ and $\mathrm{C}$ occur at the western and eastern limits of the merging line respectively and may be explained by the assumptions we have made in deriving the expected location of the merging line (length of merging line and it being symmetrical about $0 \mathrm{kV}$ potential line). Exception $\mathrm{A}$ is harder to explain, IMF By control of the meridian of the throat region would favor the Northern Hemisphere, not the Southern Hemisphere, seeing the flow across the separatrix persisting further in to the afternoon cell.

We observe flow crossing the separatrix for nearly all of the $4 \mathrm{~h}$ observing period, thus the reconnection electric field is observed in both the throat flow region and the afternoon cell. It is only at the eastern limit of our data from the afternoon convection cell in the north (1630 UT, 1230 MLT) and in the morning cell in the south (1330 UT, 1000 MLT) that this flow tends to zero. Observations made directly into the throat flow region (largest poleward flow component, Goose Bay only) or in the early afternoon cell (westward flow with frequent poleward excursions, Goose Bay and Halley) show the largest $\mathrm{E}_{\text {rec }}$. One exception to this is a short interval of flow observed at Halley (1628 UT) associated with a brief change in the sense of IMF By. The trend of $\mathrm{E}_{\text {rec }}$ towards lower values as the meridian rotates further in to the afternoon cell, seen clearly in the Goose Bay data, is consistent with the gradient of $E_{r e c}$ across the merging line predicted by Crooker (1979). However, there are space/time ambiguities in our data (we do not observe the whole ionospheric footprint of the reconnection line) which mean we cannot be certain about this.

When the $\mathrm{E}_{\text {rec }}$ value determined in the throat region (Goose Bay, 1310-1340 UT) is extrapolated to $3.5 \mathrm{~h}$ of
Table 1. The rows labelled "MODEL" give the MLT location of the $0 \mathrm{kV}$ potential line at $70^{\circ}$ latitude given by Heppner and Maynard (1987) models DE and BC. Figures in brackets give the extent of the ionospheric merging line, assuming width of $3.5 \mathrm{~h}$ centred about $0 \mathrm{kV}$ potential line. The rows labelled "OBSERVED" give observations of flow across the separatrix in the radar data

\begin{tabular}{ll}
\hline Hemisphere & By negative \\
\hline North-model & 1000 MLT (0815-1145) \\
North-observed & $0910-0949$ MLT
\end{tabular}

South-model

South-observed
1130 MLT (0945-1315)

Separatrix flow tends to zero 0930-1010 MLTmorning cell
By positive

\author{
1130 MLT (0945-1315) \\ 1010-1230 MLT \\ Contrary observation: \\ flows tend to zero in 1230-1300 \\ MLT-afternoon cell \\ 1000 MLT (0815-1145) \\ 1020-1330 MLT
}


MLT (Fig. 4) it corresponds to a large percentage of the CPD determined from the AMIE procedure. The possible gradient in the reconnection field across the throat flow region means we are likely to be over estimating the potential observed. However, Baker et al. (1997) determined the reconnection electric field potential difference across $2.6 \mathrm{~h}$ of MLT and found, when observing the same region, that it was close to $100 \%$ of the CPD.

The bursty nature of $\mathrm{E}_{\text {rec }}$ is in agreement with the numerous reports of cusp region flow bursts during $\mathrm{Bz}$ southward, but in our data is conditioned largely by the plasma flow angle and not by the magnitude of the plasma flow. This statement must be qualified by the earlier comments regards limitations of the beamswinging technique (Section 2.11) to reproduce flow bursts. Baker et al. (1997) argued that this limitation did not severely impact the results and our data confirm their discussion, by virtue of the fact that at times we measure bursts that account for $75-100 \%$ of the CPD (if the extrapolation to $3.5 \mathrm{~h}$ of MLT is valid) We also note that the swings to poleward flow are a feature seen in case studies of individual flow channel events (e.g., Pinnock et al., 1993)

\section{Conclusions}

Data from conjugate HF radars have been used to determine the latitude and orientation of the open/ closed field line boundary (the separatrix) and the dayside reconnection voltage potential difference $\left(\mathrm{E}_{\text {rec }}\right)$ at one magnetic meridian, with a time resolution of 50 or $100 \mathrm{~s}$. The results presented here are for a period of steady and strong IMF Bz southward.

The behavior of the separatrix fits theoretical expectations and observations by other instruments, giving us further confidence in the application of the HF radar technique. The cusp latitude in the two hemispheres shows the same general trend and the hemispherical displacement expected from the effects of dipole tilt. The orientation of the separatrix shows a behavior consistent with that shown by the Feldstein (1963) model of the poleward boundary of the auroral oval, except that in the Northern Hemisphere the magnitude of the tilt is much larger. We have identified factors that may contribute to the larger tilt angles but cannot quantify their effect with this data set. Our data contradict earlier claims for the influence of IMF By on the separatrix orientation. Further studies are required on what conditions the orientation of the separatrix in the cusp region.

The velocity of the separatrix was found to lie almost entirely within our error limits and as a consequence we had to use a smoothed separatrix velocity when calculation the reconnection electric field. The separatrix, with just a very few exceptions, did not move with velocities greater than $600 \mathrm{~m} \mathrm{~s}^{-1}$ in the throat flow region and $300 \mathrm{~m} \mathrm{~s}^{-1}$ in the afternoon and morning convection cells. This finding suggests that any rapid equatorward motion of the separatrix, required in some models of ionospheric signatures of FTEs, is towards the lower end of the range of published estimates. Furthermore, although our data omitted the separatrix velocity from the calculation of $\mathrm{E}_{\text {rec }}$ it still was capable of accounting for close to $100 \%$ of the CPD when observations were made from the throat flow region. This suggests that magnetic flux is primarily added to the polar cap by accelerating flows across the polar cap boundary rather than by large equatorward movement of the boundary and subsequent relaxation poleward.

The influence of IMF By on the large-scale convection pattern and the magnetic local time of the throat flow region has been demonstrated and is consistent with previous observations (Newell et al., 1989; Heppner and Maynard, 1987) and models (Cowley et al., 1991), after taking account of the frame of reference of the observations. However, we found the flow across the separatrix, in the Southern Hemisphere for IMF By positive conditions, extending further in to the afternoon cell than expected.

The day-side reconnection voltage derived confirms the earlier work of Baker et al. (1997), in that when a single radar is looking directly in to the throat flow region it can measure a large percentage $(>75 \%)$ of the CPD (as estimated by the AMIE model). This result tends to favor the recent (Crooker and Toffoletto, 1995) downward revision in estimates of the width of the ionospheric merging line, from $5 \mathrm{~h}$ to $3.5 \mathrm{~h}$ of MLT. The reconnection voltage in the throat flow region was very bursty in nature, consistent with case studies of cusp flows, and suggests that it is primarily conditioned by FTEs. As the Goose Bay radar moved in to the afternoon convection cell it observed a gradually reducing day-side reconnection voltage, eventually declining to zero potential by 1230 MLT, consistent with the predictions of the anti-parallel merging model (Crooker, 1979).

Future studies will use vectors from the SuperDARN network of HF radars (Greenwald et al., 1995), derived from dual radar operation. The network of SuperDARN radars also have the potential to image the full width of the ionospheric merging line. Such studies should utilize the findings in this study concerning the required resolution of the data.

Acknowledgements. We acknowledge the help of J. Aguda in preparing the data for analysis. The IMP-8 data was obtained form NSSDC. The College magnetogram was supplied by WDC2, Kyoto. Support for the Goose Bay radar is provided in part by NSF grant ATM-9502993 and in part by NASA grant NAG51099. The Halley radar was developed under support from the UK NERC and NSF Division of Polar Programs, grant DPP-8822172. The authors wish to acknowledge the GEM Workshop meetings for promoting this campaign period and for providing such a stimulation environment for science.

Topical Editor D. Alcaydé thanks G.T. Blanchard and P.E. Sandholt for their help in evaluating this paper.

\section{References}

Andre, R. C., Hanuise, J-P. Villain, and J-C. Cerisier, HF-radars: Multifrequency study of refraction effects and localization of scattering, Radio Sci., 32, 153, 1997. 
Baker, K. B., R. A. Greenwald, J. M. Ruohoniemi, J. R. Dudeney, M. Pinnock, and N. Mattin, PACE: Polar Anglo American Conjugate Experiment, Eos, 70 (34), 785, 1989.

Baker, K. B., and S. Wing, A new magnetic coordinate system for conjugate studies at high latitude. J. Geophys. Res., 94, 9139, 1989.

Baker, K. B., R. A. Greenwald, J. M. Ruohoniemi, J. R. Dudeney, M. Pinnock, P. T. Newell, M. E. Greenspan, and C.-I. Meng, Simultaneous HF-radar and DMSP observations of the cusp, Geophys. Res. Lett., 17, 1869, 1990.

Baker, K. B., J. R. Dudeney, R. A. Greenwald, M. Pinnock, P. T. Newell, A. S. Rodger, N. Mattin, and C.-I. Meng, HF radar signatures of the cusp and low-latitude boundary layer, J. Geophys. Res., 100, 7671, 1995.

Baker, K. B., A. S. Rodger, and G. Lu, HF-Radar observations of the rate of magnetic merging: a GEM boundary layer campaign study, J. Geophys. Res., 102, 9603, 1997.

Choe, J. Y., and D. B. Beard, The compressed geomagnetic field as a function of dipole tilt, Planet. Space Sci., 22, 595, 1974.

Coroniti, F. V., and C. F. Kennel, Can the ionosphere regulate magnetospheric convection?, J. Geophys. Res., 78, 2837, 1973.

Cowley, S. W. H., and M. Lockwood, Excitation and decay of solar-wind driven flows in the magnetosphere-ionosphere system, Ann. Geophys. 10, 103, 1992.

Cowley, S. W. H., J. P. Morelli, and M. Lockwood, Dependence of convective flows and particle precipitation in the high-latitude dayside ionosphere on the $\mathrm{X}$ and $\mathrm{Y}$ components of the interplanetary magnetic field, J. Geophys. Res., 96, 5557, 1991.

Crooker, N. U., Dayside merging and cusp geometry, J. Geophys. Res., 84, 951, 1979.

Crooker, N. U., and F. R. Toffoletto, Global aspects of magnetopauseionosphere coupling: review and synthesis, physics of the Magnetopause. Ed. P. Song, B. U. O. Sonnerup and M. F. Thomsen, AGU Monograph 90, 363, 1995.

Feldstein, Y. I., On morphology of auroral and magnetic disturbances at high latitudes, Geomag. Aeron., 3, 183, 1963.

Freeman, M. P., J. M. Ruohoniemi, and R. A. Greenwald, The determination of time-stationary two dimensional convection patterns with single-station radars, J. Geophys. Res., 96, 15 735, 1991.

Greenwald, R. A., K. B. Baker, J. M. Ruohoniemi, J. R. Dudeney, M. Pinnock, N. Mattin, J. M. Leonard, and R. P. Lepping, Simultaneous conjugate observations of dynamic variations in high-latitude convection due to changes in IMF By, J. Geophys. Res., 95, 8057, 1990.

Greenwald R. A., K. B. Baker, J. R. Dudeney, M. Pinnock, T. B. Jones, E. C. Thomas, J-P. Villain, J-C. Cerisier, C. Senior, C. Hanuise, R. D. Hunsucker, G. Sofko, J. Koehler, E. Nielsen, R. Pellinen, A. D. M. Walker, N. Sato, and H. Yamagishi, DARN/ SuperDARN: a global view of the dynamics of high latitude convection, Space Sci. Rev., 71, 761, 1995.

Heppner, J. P., and N. C. Maynard, Empirical high-latitude electric field models, J. Geophys. Res., 92, 4467, 1987.

Holzworth, R. H., and C.-I. Meng, Mathematical representation of the auroral oval, Geophys. Res. Lett., 2, 377, 1975.

Kivelson, M. G., and C. T. Russell (Eds), Introduction to Space Physics, Cambridge University Press, Cambridge, UK, 1995.

Lockwood, M., and M. N. Wild, On the quasi-periodic nature of flux transfer events, J. Geophys. Res., 98, 5935, 1993.

Lockwood, M., J. Moen, S. W. H. Cowley, A. D. Farmer, U. P. Lovhaug, H. Lühr, and V. N. Davda, Variability of dayside convection and motions of the cusp/cleft aurora, Geophys. Res. Lett., 20, 1011, 1993a.

Lockwood, M., W. F. Denig, A. D. Farmer, V. N. Davda, S. W. H. Cowley, and H. Lühr, Ionospheric signature of pulsed reconnection at the Earth's magnetopause, Nature, 361, 424, 1993 b.

Maynard, N. C., W. F. Denig, and W. J. Burke, Mapping ionospheric convection patterns to the magnetosphere, J. Geophys. Res., 100, 1713, 1995.

Meng, C-I., Latitudinal variation of the polar cusp during a geomagnetic storm, Geophys. Res. Lett., 9, 60, 1982.

Minow, J., PhD Thesis, University of Alaska, USA, 1996.

Moen J., D. Evans, H. C. Carlson, and M. Lockwood, Dayside moving auroral transients related to LLBL dynamics, Geophys. Res. Lett., 23, 3247, 1996.

Newell, P. T., and C-I. Meng, Dipole tilt angle effects on the latitude of the cusp and cleft/low latitude boundary layer, J. Geophys. Res., 94, 6949, 1989.

Newell, P. T., C-I Meng, D. G. Sibeck, and R. P. Lepping, Some low altitude cusp dependencies on the Interplanetary Magnetic Field, J. Geophys. Res., 94, 8921, 1989.

Pinnock, M., A. S. Rodger, J. R. Dudeney, K. B. Baker, R. A. Greenwald, and M. Greenspan, Observations of an enhanced convection channel in the cusp ionosphere, J. Geophys. Res., 98, 3767, 1993.

Pinnock, M., A. S. Rodger, J. R. Dudeney, F. Rich, and K. B. Baker, High spatial and temporal resolution observations of the ionospheric cusp, Ann. Geophys, 13, 919, 1995.

Pudovkin, M. I., S. A. Zaitseva, P. E. Sandholt, and A. Egeland, Dynamics of aurorae in the cusp region and characteristics of magnetic reconnection at the magnetopause, Planet. Space Sci., 40, 879, 1992.

Richmond, A. D., and Y. Kamide, Mapping electrodynamic features of the high-latitude ionosphere from localized observations: technique, J. Geophys. Res., 93, 5741, 1988.

Rodger, A. S., S. B. Mende, T. J. Rosenberg, and K. B. Baker, Simultaneous optical and HF radar observations of the ionopsheric cusp, Geophys. Res. Lett., 22, 2045, 1995.

Rodger, A. S., and M. Pinnock, The ionospheric response to flux transfer events: the first few minutes, Ann. Geophys, 15, 685, 1997.

Rodger, A. S. Ionospheric signatures of magnetopause processes, in Polar cap boundary phenomena, Ed. J. Moen, Kluwer Academic Publishers, p 115, 1998.

Ruohoniemi, J. M., R. A. Greenwald, J-P. Villian, K. B. Baker, and C Hanuise, Mapping high-latitude plasma convection with coherent HF-radars, J. Geophys. Res., 94, 13 463, 1989.

Sandholt, P. E., C. J. Farrugia, L. F. Burlaga, J. A. Holtet, J. Moen, B. Lybekk, B. Jacobsen, D. Opsvik, A. Egeland, R. Lepping, A. J. Lazarus, T. Hansen, A. Brekke, and E. FriisChristensen, Cusp/Cleft auroral activity in relation to solar wind dynamic pressure, interplanetary magnetic field $\mathrm{Bz}$ and $\mathrm{By}$, J. Geophys. Res., 99, 17323, 1994.

Siscoe G. L., and T. S. Huang, Polar cap inflation and deflation, J. Geophys. Res., 90, 543, 1985.

Tsganenko, N. A., Methods for quantitative modelling of the magnetic field from Birkeland currents, Planet. Space Sci., 39, 641, 1991.

Villain J-P., G. Caudal, and C. Hanuise, A SAFARI-EISCAT comparison between the velocity of $\mathrm{F}$ region small-scale irregularities and the ion drift., J. Geophys. Res., 90, 8433, 1985. 